\title{
Testing temperature-induced proteomic changes in the plant-associated bacterium Pseudomonas fluorescens SBW25
}

\author{
C. G. Knight, ${ }^{1,2,3 *}$ X. X. Zhang, ${ }^{1,4,5}$ A. Gunn, ${ }^{4}$ \\ T. Brenner, ${ }^{3+}$ R. W. Jackson, ${ }^{1 \$}$ S. R. Giddens, ${ }^{1}$ \\ S. Prabhakar, ${ }^{3}$ N. Zitzmann ${ }^{3}$ and P. B. Rainey ${ }^{1,4,5}$ \\ ${ }^{1}$ Department of Plant Sciences, University of Oxford, \\ South Parks Road, Oxford OX1 3RB, UK. \\ ${ }^{2}$ Faculty of Life Sciences, The University of Manchester, \\ Oxford Road, Manchester M13 9PT, UK. \\ ${ }^{3}$ Oxford Glycobiology Institute, Department of \\ Biochemistry, University of Oxford, South Parks Road, \\ Oxford OX1 3QU, UK. \\ ${ }^{4} \mathrm{New}$ Zealand Institute for Advanced Study and ${ }^{5}$ Allan \\ Wilson Centre for Molecular Ecology and Evolution, \\ Massey University, Private Bag 102904, North Shore \\ Mail Centre 0745, Auckland, New Zealand.
}

\section{Summary}

Traits used by bacteria to enhance ecological performance in natural environments are not well understood. Recognizing that the saprophytic plant-colonizing bacterium Pseudomonas fluorescens SBW25 experiences temperatures in its natural environment significantly cooler than the $28^{\circ} \mathrm{C}$ routinely used in the laboratory, we identified proteins differentially expressed between $28^{\circ} \mathrm{C}$ and the more environmentally relevant temperature of $14^{\circ} \mathrm{C}$. Of 2102 protein isoforms, 32 were temperature responsive and identified by mass spectrometry. Seven of these (OmpR, MucD, GuaD, OsmY and three of unknown function, Tee1, Tee2 and Tee3) were selected for genetic and ecological analyses. In each instance, changes in protein expression with temperature were mirrored by parallel transcriptional changes. The fitness contribution of the genes encoding each of the seven proteins was larger at $14^{\circ} \mathrm{C}$ than $28^{\circ} \mathrm{C}$ and included two cases of trade-offs (enhanced fitness at one temperature and reduced fitness at the other - mucD and tee2 deletions). The relationship

\footnotetext{
Received 27 April, 2009; accepted 7 October, 2009. *For correspondence. E-mail chris.knight@manchester.ac.uk; Tel. (+44) 161 2755378. Present address: 'Department of Clinical Neurology, University of Oxford, John Radcliffe Hospital, Headley Way, Oxford OX3 9DU, UK; §School of Biological Sciences, The University of Reading, Whiteknights, Reading RG6 6AJ, UK.
}

C 2009 Society for Applied Microbiology and Blackwell Publishing Ltd between the fitness effects of genes in vitro and in vivo was variable, but two temperature-responsive genes - osm $Y$ and $m u c D$ - contribute substantially to the ability of $P$. fluorescens to colonize the plant environment.

\section{Introduction}

The plant-colonizing Pseudomonas, including P. fluorescens, P. putida and $P$. syringae, are typically grown in the laboratory environment at $28^{\circ} \mathrm{C}$. While maximizing growth rate, such high temperatures are unlikely to be encountered in the wild. Given the tendency of bacteria to tailor expression of ecologically relevant traits to the environment in which they are required (Rainey, 1999; Ramos et al., 2001; Gal et al., 2003), the existence of a subset of traits expressed in response to ambient soil temperatures seems probable. In $P$. fluorescens strain MF1, nearly $40 \%$ of $>800$ transcriptional fusion constructs randomly inserted into the genome were thermally regulated (Regeard et al., 2000) with reduced temperature being associated with increasing expression and activity of exported phosphatases, proteases and lipases (Gugi et al., 1991; Burini et al., 1994). Indeed in plant-associated bacteria more generally, expression of genes involved in pathogenicity is associated with growth at temperatures below the growth optimum (Smirnova et al., 2001; Ullrich, 2002). In P. fluorescens, such low-temperature gene expression effects have been associated with changes in physicochemical adhesion properties (Hemery et al., 2007) and increased colonization ability in different environments (Seong et al., 1991; Picot et al., 2004).

Understanding environmentally responsive gene regulation requires data across biological levels, from genotype, via the cellular phenotypes of transcription and protein levels, to organismal fitness. It also requires data across controlled in vitro environments and more realistic in vivo environments. Here, we make these connections for a sample of temperature-responsive genes in $P$. fluorescens SBW25, a sequenced (Silby et al., 2009) plant growth-promoting bacterium that, like other plantassociated Pseudomonas species, can colonize the rhizosphere and phyllosphere of a range of plants. 
Table 1. Temperature-responsive proteins chosen for detailed study.

\begin{tabular}{lll}
\hline & & $\begin{array}{l}\text { Temperature } \\
\text { with } \\
\text { increased } \\
\text { expression } \\
\left({ }^{\circ} \mathrm{C}\right)\end{array}$ \\
$\begin{array}{l}\text { Protein (and } \\
\text { associated orf) }\end{array}$ & Putative identification & 14 \\
\hline OsmY (Pflu5248) & Periplasmic protein osmY & 14 \\
Tee1 (Pflu2044) & Periplasmic solute-binding protein & 14 \\
Tee2 (Pflu0824) & Porin & 14 \\
Tee3 (Pflu5795) & Hypothetical protein & 28 \\
GuaD (Pflu4595) & Guanine deaminase & 28 \\
MucD (Pflu1471) & Serine protease & 28 \\
OmpR (Pflu0259) & Transcriptional regulator & \\
\hline
\end{tabular}

Our study begins with a differential proteomic analysis of the functional molecules, namely proteins, and then proceeds to link changes in protein expression with changes in both transcription and fitness. The revealed relationships are complex, but nonetheless validate our conjecture that genes expressed at 'environmentally relevant' temperatures contribute towards the success of $P$. fluorescens SBW25 in nature.

\section{Results and discussion}

Identification of temperature-sensitive protein expression by differential proteomics

Two-dimensional (2D) gel electrophoresis resolved 2102 protein spots in wild-type $P$. fluorescens SBW25, of which 56 were differentially expressed between $14^{\circ} \mathrm{C}$ and $28^{\circ} \mathrm{C}$ (Figs S1 and S2, Table S1). Thirty-two of these spots were identified by mass spectrometry as corresponding to one of 31 single open reading frames in the genome sequence (Tables S1 and S2). The identified proteins fall into a range of predicted functional categories, which is expected given that changes may include both direct temperature responses and indirect effects, e.g. effects via changes in membrane fluidity or growth rate. The temperature-responsive proteins include several chaperones (HtpG, DnaK, CspA and Cpn10 and GroEL), a small minority of predicted membrane proteins (notably OprG) and six conserved but hitherto hypothetical proteins.

Seven proteins differentially expressed between temperatures were selected for transcriptional and mutational analysis (Table 1). This sample was chosen with a view to including a balance of up- and down-regulated proteins and proteins with a range of predicted functions; preference was given to proteins with unambiguous identifications (Table S1) and where prior information suggested possible roles in plant-microbe interactions or thermoregulation. Four of the chosen proteins are characterized: MucD, GuaD, OmpR, OsmY, and three are of unknown function: Tee1, Tee2 and Tee3. Specifically, MucD is a putative serine protease, required for resis- tance to high temperatures and oxidative stress (Boucher et al., 1996). Guanine deaminase (GuaD) is a ubiquitous enzyme that catalyses the hydrolytic deamination of guanine into xanthine, the first step of purine metabolism (Fernandez et al., 2009). Significantly, expression of guaD in P. fluorescens SBW25 is elevated during bacterial colonization of the rhizosphere (Silby et al., 2009). OmpR is the response regulator of the two-component regulatory system OmpR-EnvZ, known to regulate temperaturedependent lipase secretion in $P$. fluorescens (McCarthy et al., 2004). OsmY is a putative periplasmic protein with two phospholipid-binding domains. In Escherichia coli, expression of $o s m Y$ is induced in stationary phase and osmotically regulated (Yim et al., 1994). The three genes of unknown function are designated tee1, tee2 and tee3, respectively, because of their temperature expression effect. tee1 (pflu2044) encodes a putative solute-binding protein of an ABC-type transporter. tee2 (pflu0824) is predicted to encode a porin belonging to the OprD family. Interestingly, two isoforms of Tee2 plus an adjacent protein (Pflu0823) were identified in the proteomic analysis (Table S2). tee3 is highly conserved across Pseudomonadales, with a single copy (at least $83 \%$ amino acid identity) in each of the 19 Pseudomonas strains with complete genomes available, plus the sister taxon Azotobacter vinelandii [PseudoDB version 22 (Chaudhuri and Pallen, 2006)]. PFAM (Finn et al., 2008) identifies similarity to an $S$-adenosylmethionine (SAM)-dependent methyltransferase domain (Table S3). Proteins with this fold are widespread, with varied biological functions (Martin and McMillan, 2002), including a role in plant colonization in $P$. syringae (Braun et al., 2008). Among the seven selected proteins, three (MucD, GuaD and OmpR) have increased expression at $28^{\circ} \mathrm{C}$ and four (OsmY, Tee1, Tee2 and Tee3) have the greater expression at $14^{\circ} \mathrm{C}$ (Table 1).

\section{Temperature-sensitive protein expression corresponds in part to temperature-sensitive transcription}

Transcriptional expression of the selected genes was examined using chromosomally integrated promoterless 'lacZ fusions; however, a 'lacZ fusion to tee2 proved impossible to generate. $\beta$-Galactosidase activities were measured for cells grown at both $14^{\circ} \mathrm{C}$ and $28^{\circ} \mathrm{C}$ and showed significant variation in their response to temperature (Table S4A). For all genes tested the temperature at which protein expression was the greatest was also the temperature at which lac $Z$ expression was the greatest (as indicated by the fact that the two colours in each point in Fig. 1 are similar; see also Fig. S3). Overall, there is the suggestion of a positive relationship between changes seen in transcription and changes seen in protein levels (Fig. S3; Table S5, rank correlation $r=0.80, P=0.056$, $n=6)$. However, transcription alone does not fully explain 


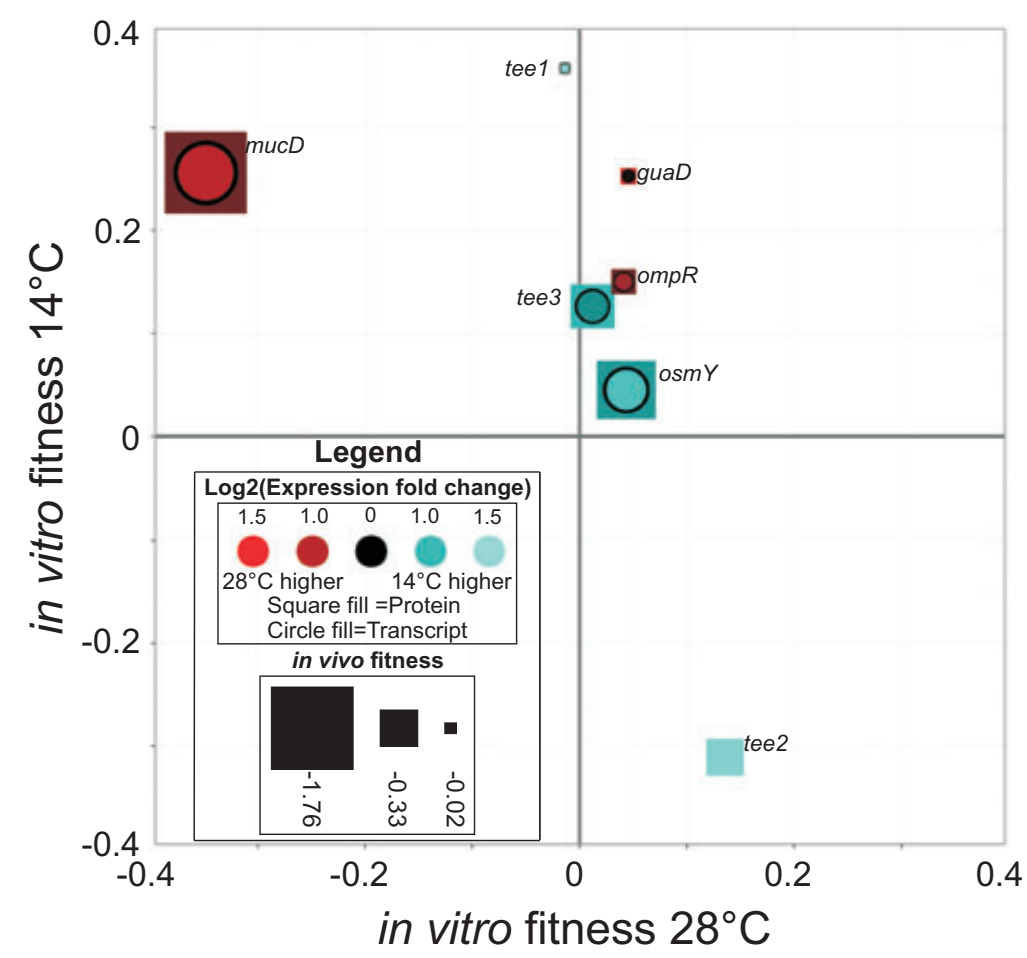

Fig. 1. Expression and competitive fitness effects of temperature-responsive genes in different environments. Location indicates the competitive fitness (selection rate constant, SRC, $r$, units $\mathrm{h}^{-1}$ ) in vitro of the strain with the gene deleted. Values above zero indicate increased fitness relative to the marked wild-type strain and values below zero indicate decreased fitness. The size of the square indicates the absolute SRC in the plant environment (average of shoot and root since these are non-significantly different; all values are either negative or non-significantly different from zero). For ease of comparison, the SRC scales have been offset such that the wild type appears equal to zero (from which it was non-significantly different). Each value is an average of 4-10 replicates. Colours indicate the fold change of expression between $28^{\circ} \mathrm{C}$ and $14^{\circ} \mathrm{C}$, the square indicating the change in protein levels, the central circle indicating change in transcription. Gene labels as in Table 1. tee2 has no transcription data as the construct proved impossible to make. tee1 has no protein fold change as it was not detectable at $28^{\circ} \mathrm{C}$. Fuller details on these data (including explicit transcription and protein levels with error bars and separation according to shoot and root with errors) are given in supporting Figs S3 and S4; materials and experimental procedures are given in Appendix S1 and supporting Tables S6 and S7.

the differences in protein levels (e.g. guaD shows a relatively small, 1.2-fold, change in transcription, but a large, 2.1-fold, change in amount of the protein present at the higher temperature; Fig. 1).

\section{Fitness effects of temperature-responsive genes are environment specific}

Seven in-frame deletion mutants were constructed for the selected temperature-responsive genes. In each instance this involved deletion of a single gene; however, for tee2, which is part of a 10-gene operon (pflu0819-pflu0828) from which two isoforms of Tee2 and the gene product of pflu0823 were identified by proteomic analysis (Tables S1 and S2), the entire operon was deleted. The resulting seven mutants were each competed against a neutrally marked ancestor (Zhang and Rainey, 2007) in similar in vitro conditions to those used for the proteomics.

Despite selecting genes with measurable, responsive expression and using a sensitive measure of ecological performance (relative fitness) the effect of gene deletion under standard laboratory conditions $\left(28^{\circ} \mathrm{C}\right.$, rich medium) was close to zero in all cases except mucD ( $x$-axis Fig. 1, see also Fig. S4A). This is consistent with large-scale surveys in model organisms where most single-gene deletions do not affect phenotype in the laboratory environment. For example, a study of bakers' yeast showed that $19 \%$ of genes are essential, while a further $15 \%$ showed a growth phenotype under standard laboratory conditions (Giaever et al., 2002).

Various forms of 'buffering' or even a selective advantage to robustness against mutation have been posited to explain such small phenotypic effects of gene deletion (Wagner, 2000; Hartman et al., 2001). However, a more likely general explanation is that apparent robustness to gene deletion is a by-product of adaptation in other environments (Papp et al., 2009). We therefore repeated the in vitro competitions at the more environmentally relevant temperature of $14^{\circ} \mathrm{C}$ ( $y$-axis, Fig. 1 , see also Fig. S4A). Consistent with the idea that relevant phenotypes should 
become apparent at a more environmentally relevant temperature, the absolute magnitude of the fitness effect of each gene deletion was typically greater at $14^{\circ} \mathrm{C}$ than $28^{\circ} \mathrm{C}$ (i.e. with the exception of $m u c D$, all the points in Fig. 1 are closer to the $y$-axis than the $x$-axis). This temperature effect on the fitness of deletion strains was significant and varied significantly among strains (ANOVA, $P<0.05$ in each case, Table S4B). However, several genes showed a cost to carriage at $14^{\circ} \mathrm{C}$ (notably guaD and tee1).

\section{OsmY and MucD contribute most to fitness in the plant environment}

To test the hypothesis that temperature-responsive genes contribute to the ecological performance of $P$. fluorescens in the plant environment, each deletion mutant was competed against a neutrally marked wild-type strain on sugar beet seedlings (indicated by square size in Fig. 1, see also Fig. S4B). There was significant variation in fitness among strains (ANOVA, $P<0.0001$, Table S4C). In all cases, the estimated fitness effect of possessing the gene in the seedling environment was either positive or approximately zero. Interestingly, two of the characterized genes, guaD and ompR, have effectively zero effect in the plant environment, despite guaD being specifically switched on in the root environment (Silby et al., 2009). The two temperature-responsive genes that contribute most substantially to fitness in the plant environment are osm $Y$ and mucD (illustrated by being the largest squares in Fig. 1).

OsmY, while contributing little to fitness in vitro, has a major effect in the plant environment (Fig. 1). Its expression - on both protein and gene levels - was upregulated at reduced temperature (Fig. 1). In E. coli, osm $Y$ has complex transcriptional regulation (Lange et al., 1993; Yim et al., 1994), including upregulation by both the stationary-phase sigma factor RpoS and reduced temperatures (Rajkumari and Gowrishankar, 2001). In P. fluorescens, the RpoS regulon has been implicated in stress tolerance in vitro and also in plant colonization in the field (Stockwell and Loper, 2005). Our data show little fitness effect of the gene in vitro, but reveal a strong benefit to carrying the gene in vivo (Fig. 1). This makes osm Y a strong candidate for an effector of RpoS-dependent plant colonization.

MucD has the largest fitness contribution seen here in the plant environment (Fig. 1). In Pseudomonas aeruginosa MucD is a negative regulator of alginate biosynthesis and a homologue of the E. coli protein HtrA (DegP): a serine protease that degrades misfolded and damaged proteins at higher temperatures $\left(>22^{\circ} \mathrm{C}\right)$, with independent chaperone activity at lower temperatures (Spiess et al., 1999). In Pseudomonas MucD is necessary for pathogenicity in both animals ( $P$. aeruginosa) and plants ( $P$. syringae and $P$. aeruginosa) (Yorgey et al., 2001), with a separate role in alginate regulation (Yorgey et al., 2001; Wood and Ohman, 2006). It is also upregulated by exposure to root exudates in P. putida (Cheng et al., 2009). Unsurprisingly, given MucD's protection against thermally induced protein damage, we find $P$. fluorescens SBW25 MucD upregulated at the higher temperature (Fig. 1).

The precise causes of the deleterious fitness effects in the mucD mutant are unclear; however, they may reflect a role in the expression of cellulose. In P. fluorescens SBW25, the principal extracellular matrix implicated in ecological performance in the plant environment, as well as in biofilm formation, is a cellulose-like polymer rather than alginate, the expected regulatory target of MucD (Spiers et al., 2002; Gal et al., 2003). Nonetheless, the cellulose-encoding wss operon is subjected to regulation by known alginate regulators [AlgR and AmrZ (AlgZ) (Giddens et al., 2007)] and MucD has been independently identified as necessary for certain regulatory pathways of surface colonization involving the overproduction of the cellulose-like polymer (McDonald et al., 2009), suggesting a mechanism for its fitness effect in the plant environment.

\section{Trade-offs in fitness effects across temperature}

The striking pleiotropic effects associated with deletion of mucD and tee2 warrant comment (Fig. 1). In both cases, deletion of the gene positively affects fitness at one temperature, but negatively affects fitness at the other. For example, a functional copy of mucD is clearly advantageous at $28^{\circ} \mathrm{C}$ (deletion of $m u c D$ causes a large reduction in fitness at $28^{\circ} \mathrm{C}$ ), but at $14^{\circ} \mathrm{C}$ mucD appears to be costly (deletion of the gene results in a significant fitness increase at $14^{\circ} \mathrm{C}$ ). tee 2 shows similar antagonistic pleiotropy across temperatures, although in the opposite direction to mucD (i.e. tee2 is in the lower right quadrant of Fig. 1, whereas mucD is in the upper left). In both cases, the environment in which fitness is most enhanced by presence of the gene is the environment in which expression was maximal (Fig. 1). While trade-offs may be a general feature of low temperature adaptation (Bennett and Lenski, 2007), such clear examples of fitness trade-offs due to single genes are unexpected and point to the fact that expression of mucD and tee2 is occurring at temperatures where complete repression would seem to be beneficial. While the molecular bases of these tradeoffs are unknown, their study is likely to shed light on factors that constraint ecological performance of bacteria in nature.

\section{Conclusion}

Many different approaches can be used to study the fit between organism and environment. Here we have taken 
a proteomic approach that provides insight into phenotype at the level of the critical functional components, namely the proteins. In this instance, our focus has been that set of proteins differentially expressed in response to temperature. Rather than simply characterize individual proteins, we have sought to integrate this information into both lower (transcription) and higher (fitness) levels of biological organization; moreover, to place this knowledge in an ecologically relevant context. The challenges of such an approach are considerable, and - as is evident in this work - interrelationships between expression, environment and fitness can be complex and not always in accord with simple models. However, such complexity is likely a true reflection of biological reality that will ultimately deliver improved insight into the lives of bacteria in natural environments.

\section{Acknowledgements}

We thank Hao Chang for technical assistance. C.G.K. is supported by a Wellcome Trust Research Career Development Fellowship. Some of the work was carried out under a grant from NERC's environmental genomics programme.

\section{References}

Bennett, A.F., and Lenski, R.E. (2007) An experimental test of evolutionary trade-offs during temperature adaptation. Proc Natl Acad Sci USA 104 (Suppl. 1): 8649-8654.

Boucher, J.C., Martinez-Salazar, J., Schurr, M.J., Mudd, M.H., Yu, H., and Deretic, V. (1996) Two distinct loci affecting conversion to mucoidy in Pseudomonas aeruginosa in cystic fibrosis encode homologs of the serine protease HtrA. J Bacteriol 178: 511-523.

Braun, S.D., Volksch, B., Nuske, J., and Spiteller, D. (2008) 3-Methylarginine from Pseudomonas syringae pv. syringae $22 \mathrm{~d} / 93$ suppresses the bacterial blight caused by its close relative Pseudomonas syringae pv. glycinea. Chembiochem 9: 1913-1920.

Burini, J.F., Gugi, B., Merieau, A., and Guespin-Michel, J.F. (1994) Lipase and acidic phosphatase from the psychrotrophic bacterium Pseudomonas fluorescens: two enzymes whose synthesis is regulated by the growth temperature. FEMS Microbiol Lett 122: 13-18.

Chaudhuri, R.R., and Pallen, M.J. (2006) xBASE, a collection of online databases for bacterial comparative genomics. Nucleic Acids Res 34: D335-D337.

Cheng, Z., Duan, J., Hao, Y., McConkey, B.J., and Glick, B.R. (2009) Identification of bacterial proteins mediating the interactions between Pseudomonas putida UW4 and Brassica napus (Canola). Mol Plant Microbe Interact 22: 686694.

Fernandez, J.R., Byrne, B., and Firestein, B.L. (2009) Phylogenetic analysis and molecular evolution of Guanine deaminases: from Guanine to dendrites. J Mol Evol 68: 227-235.

Finn, R.D., Tate, J., Mistry, J., Coggill, P.C., Sammut, S.J., Hotz, H.R., et al. (2008) The Pfam protein families database. Nucleic Acids Res 36: D281-D288.
Temperature-responsive expression in Pseudomonas

Gal, M., Preston, G.M., Massey, R.C., Spiers, A.J., and Rainey, P.B. (2003) Genes encoding a cellulosic polymer contribute toward the ecological success of Pseudomonas fluorescens SBW25 on plant surfaces. Mol Ecol 12: 31093121.

Giaever, G., Chu, A.M., Ni, L., Connelly, C., Riles, L., Veronneau, S., et al. (2002) Functional profiling of the Saccharomyces cerevisiae genome. Nature 418: 387-391.

Giddens, S.R., Jackson, R.W., Moon, C.D., Jacobs, M.A., Zhang, X.X., Gehrig, S.M., and Rainey, P.B. (2007) Mutational activation of niche-specific genes provides insight into regulatory networks and bacterial function in a complex environment. Proc Natl Acad Sci USA 104: 18247-18252.

Gugi, B., Orange, N., Hellio, F., Burini, J.F., Guillou, C., Leriche, F., and Guespin-Michel, J.F. (1991) Effect of growth temperature on several exported enzyme activities in the psychrotrophic bacterium Pseudomonas fluorescens. J Bacteriol 173: 3814-3820.

Hartman, J.L., Garvik, B., and Hartwell, L. (2001) Principles for the buffering of genetic variation. Science 291: 1001-1004.

Hemery, G., Chevalier, S., Bellon-Fontaine, M.N., Haras, D., and Orange, N. (2007) Growth temperature and OprF porin affect cell surface physicochemical properties and adhesive capacities of Pseudomonas fluorescens MF37. J Ind Microbiol Biotechnol 34: 49-54.

Lange, R., Barth, M., and Hengge-Aronis, R. (1993) Complex transcriptional control of the sigma s-dependent stationaryphase-induced and osmotically regulated osmY (csi-5) gene suggests novel roles for Lrp, cyclic AMP (cAMP) receptor protein-CAMP complex, and integration host factor in the stationary-phase response of Escherichia coli. J Bacteriol 175: 7910-7917.

McCarthy, C.N., Woods, R.G., and Beacham, I.R. (2004) Regulation of the aprX-lipA operon of Pseudomonas fluorescens $\mathrm{B} 52$ : differential regulation of the proximal and distal genes, encoding protease and lipase, by ompRenvZ. FEMS Microbiol Lett 241: 243-248.

McDonald, M.J., Gehrig, S.M., Meintjes, P.L., Zhang, X., and Rainey, P.B. (2009) Adaptive divergence in experimental populations of Pseudomonas fluorescens IV. Genetic constraints guide evolutionary trajectories in a parallel adaptive radiation. Genetics 183: 1041-1053.

Martin, J.L., and McMillan, F.M. (2002) SAM (dependent) I AM: the S-adenosylmethionine-dependent methyltransferase fold. Curr Opin Struct Biol 12: 783-793.

Papp, B., Teusink, B., and Notebaart, R.A. (2009) A critical view of metabolic network adaptations. HFSP $J$ 3: 2435.

Picot, L., Mezghani-Abdelmoula, S., Chevalier, S., Merieau, A., Lesouhaitier, O., Guerillon, J., et al. (2004) Regulation of the cytotoxic effects of Pseudomonas fluorescens by growth temperature. Res Microbiol 155: 39-46.

Rainey, P.B. (1999) Adaptation of Pseudomonas fluorescens to the plant rhizosphere. Environ Microbiol 1: 243-257.

Rajkumari, K., and Gowrishankar, J. (2001) In vivo expression from the RpoS-dependent P1 promoter of the osmotically regulated proU operon in Escherichia coli and Salmonella enterica serovar Typhimurium: activation by rho and hns mutations and by cold stress. J Bacteriol 183: 6543-6550. 
Ramos, J.L., Gallegos, M.T., Marques, S., Ramos-Gonzalez, M.I., Espinosa-Urgel, M., and Segura, A. (2001) Responses of Gram-negative bacteria to certain environmental stressors. Curr Opin Microbiol 4: 166-171.

Regeard, C., Merieau, A., and Guespin-Michel, J.F. (2000) A bioluminescence assay for screening thermoregulated genes in a psychrotrophic bacterium Pseudomonas fluorescens. J Appl Microbiol 88: 183-189.

Seong, K.-Y., Höfte, M., Boelens, J., and Verstraete, W. (1991) Growth, survival, and root colonization of plant growth beneficial Pseudomonas fluorescens ANP15 and Pseudomonas aeruginosa 7NSK2 at different temperatures. Soil Biol Biochem 23: 423-428.

Silby, M.W., Cerdeno-Tarraga, A.M., Vernikos, G.S., Giddens, S.R., Jackson, R.W., Preston, G.M., et al. (2009) Genomic and functional analyses of diversity and plant interactions of Pseudomonas fluorescens. Genome Biol 10: R51.

Smirnova, A., Li, H., Weingart, H., Aufhammer, S., Burse, A., Finis, K., et al. (2001) Thermoregulated expression of virulence factors in plant-associated bacteria. Arch Microbiol 176: 393-399.

Spiers, A.J., Kahn, S.G., Bohannon, J., Travisano, M., and Rainey, P.B. (2002) Adaptive divergence in experimental populations of Pseudomonas fluorescens. I. Genetic and phenotypic bases of wrinkly spreader fitness. Genetics 161: 33-46.

Spiess, C., Beil, A., and Ehrmann, M. (1999) A temperaturedependent switch from chaperone to protease in a widely conserved heat shock protein. Cell 97: 339-347.

Stockwell, V.O., and Loper, J.E. (2005) The sigma factor RpoS is required for stress tolerance and environmental fitness of Pseudomonas fluorescens Pf-5. Microbiology 151: 3001-3009.

Ullich, M.S. (2002) Thermoresponsive phytotoxin production in plant pathogenic bacteria. In Advances in Microbial Toxin Research and Its Biotechnological Exploitation. Upadhyay, R.K. (ed.). Berlin, Germany: Springer, pp. 81-91.

Wagner, A. (2000) Robustness against mutations in genetic networks of yeast. Nat Genet 24: 355-361.

Wood, L.F., and Ohman, D.E. (2006) Independent regulation of MucD, an HtrA-like protease in Pseudomonas aeruginosa, and the role of its proteolytic motif in alginate gene regulation. J Bacteriol 188: 3134-3137.

Yim, H.H., Brems, R.L., and Villarejo, M. (1994) Molecular characterization of the promoter of osmY, an rposdependent gene. J Bacteriol 176: 100-107.

Yorgey, P., Rahme, L.G., Tan, M.W., and Ausubel, F.M. (2001) The roles of mucD and alginate in the virulence of Pseudomonas aeruginosa in plants, nematodes and mice. Mol Microbiol 41: 1063-1076.

Zhang, X.X., and Rainey, P.B. (2007) Construction and validation of a neutrally-marked strain of Pseudomonas fluorescens SBW25. J Microbiol Methods 71: 78-81.

\section{Supporting information}

Additional Supporting Information may be found in the online version of this article:
Fig. S1. Example 2D gels at the two temperatures considered: (A) $14^{\circ} \mathrm{C}$, (B) $28^{\circ} \mathrm{C}$. The areas boxed in (A) are shown in detail in Fig. S2.

Fig. S2. Example spots identified via the differential proteomic analysis. The gel regions are those highlighted in Fig. S1A; arrows indicate two differentially expressed spots. Fig. S3. Transcript and protein expression at $14^{\circ} \mathrm{C}$ and $28^{\circ} \mathrm{C}$. Each arrow goes from the expression at $28^{\circ} \mathrm{C}$ to the expression at $14^{\circ} \mathrm{C}$. Fine lines are standard error bars. Transcription is given in units of log to the base 10 Miller units measured using chromosomally integrated promoterless 'lacZ fusions. The protein expression is given in arbitrary units based on the spot density on 2D gels. Arrows are labelled with the gene/ protein name in Table 1.

Fig. S4. Fitness of strains with temperature-responsive genes knocked out $(A)$ in vitro, $(B)$ in the plant environment. The fitness given is the selection rate constant $\left(r\right.$, units $\left.\mathrm{h}^{-1}\right)$. Values above zero indicate increased fitness relative to the marked wild-type strain and values below zero indicate decreased fitness. Each value is an average of 4-10 replicates with standard error bars. The values for the wild-type controls $(W T)$ were insignificantly different from zero $(P>0.1$, Wilcoxon signed rank test in each treatment separately or all treatments combined). For ease of comparison in each treatment the scale has been offset such that the control appears equal to zero [observed control values \pm standard error (A) $14^{\circ} \mathrm{C}=-0.10 \pm 0.19 ; 28^{\circ} \mathrm{C}=-0.13 \pm 0.17$; $(\mathrm{B})$ shoot $=$ $-0.18 \pm 0.12 ;$ root $=-0.11 \pm 0.07]$. Strains are labelled according to the gene deleted (Table 1/Table S2).

Table S1. Protein identifications. All the data for a differentially expressed spot are given in one row; in each case the information given is: locus ID from the Pseudomonas fluorescens SBW25 genome; the identified peptides corresponding to those loci; the score of the match (from Mascot); the putative identity of the protein; the observed mass and $\mathrm{pl}$ in the gels and the calculated masses and pls of the identified proteins. Where multiple identifications were obtained from a single spot, these are given in separate columns (up to 5). These correspond both to the same spot from different gels (in which case the same locus may be identified) or multiple proteins found within a single spot (in which case this is indicated in the 'multiple proteins?' column). A threshold protein score of 30 was used to indicate identification, however matches below that threshold are also shown. Table 1 principally lists identifications with a score $>50$.

Table S2. Proteins with increased expression at $14^{\circ} \mathrm{C}$ and $28^{\circ} \mathrm{C}$. Highlighted rows correspond to proteins targeted for genetic manipulation and further characterized in Table S3. Cases where a single protein was identified from a differentially expressed spot with high confidence (see Table S1) are given. All identifications from differentially expressed spots and supporting evidence are given in Table S1.

Table S3. Homology of temperature-responsive genes genetically characterized in this study

Table S4. ANOVA for transcription and fitness of knockout strains. Shown are the analyses for transcription (A), in vitro fitness (selection rate constant) (B) and in vivo fitness (selection rate constant) (C). 'Temperature' is $14^{\circ} \mathrm{C}$ versus $28^{\circ} \mathrm{C}$, 'Location' is shoot versus root. Significant effects $(P<0.05)$ are highlighted in bold. In (C), qualitatively the same results are obtained if the identity of the plant from which the root and 
shoot measurements were taken is included as a random effect or if the model is sequentially reduced to a minimal adequate model by removing the interaction and 'Location' effects.

Table S5. Correlations among variables. Pairwise Pearson correlations $(-1=$ perfect inverse correlation, $0=$ no correlation, 1 = perfect positive correlation) are given among the averages of each trait for each gene. Fold changes use a log scale. For fitness in vivo, the average of the root and shoot values is used. For Tee1, where two protein spots were identified, the average of the two protein fold changes is used. Genetic fitness effect in vitro at $28^{\circ} \mathrm{C}$ is not given since this is only distinguishable from zero for one gene $(m u c D$, see Fig. S1).

Table S6. Bacterial strains and plasmids used in this study. Table S7. Oligonucleotide primers used in this study.

Appendix S1. Experimental procedures.

Please note: Wiley-Blackwell are not responsible for the content or functionality of any supporting materials supplied by the authors. Any queries (other than missing material) should be directed to the corresponding author for the article. 